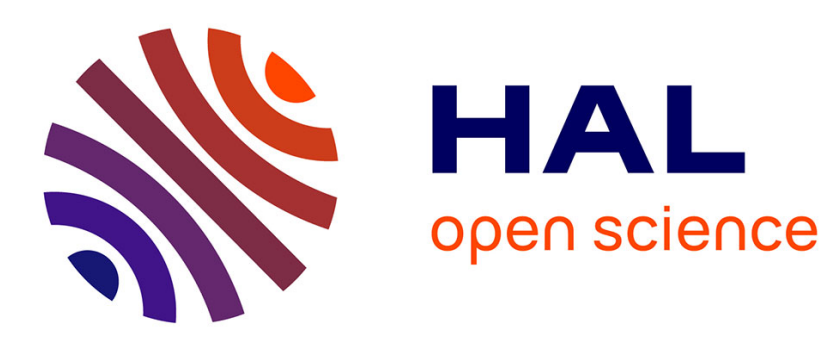

\title{
Direct measurement of the surface energy of bimetallic nanoparticles: Evidence of Vegard's rule-like dependence
} Adrian Chmielewski, Jaysen Nelayah, Hakim Amara, Jérôme Creuze, Damien Alloyeau, Guillaume Wang, Christian Ricolleau

\section{- To cite this version:}

Adrian Chmielewski, Jaysen Nelayah, Hakim Amara, Jérôme Creuze, Damien Alloyeau, et al.. Direct measurement of the surface energy of bimetallic nanoparticles: Evidence of Vegard's rule-like dependence. Physical Review Letters, 2017, 120 (2), 10.1103/PhysRevLett.120.025901 . hal-03175987

\section{HAL Id: hal-03175987 \\ https://hal.science/hal-03175987}

Submitted on 22 Mar 2021

HAL is a multi-disciplinary open access archive for the deposit and dissemination of scientific research documents, whether they are published or not. The documents may come from teaching and research institutions in France or abroad, or from public or private research centers.
L'archive ouverte pluridisciplinaire HAL, est destinée au dépôt et à la diffusion de documents scientifiques de niveau recherche, publiés ou non, émanant des établissements d'enseignement et de recherche français ou étrangers, des laboratoires publics ou privés. 


\title{
Direct measurement of the surface energy of bimetallic nanoparticles: Evidence of Vegard's rule-like dependence
}

\author{
Adrian Chmielewski, ${ }^{1}$ Jaysen Nelayah, ${ }^{1}$ Hakim Amara, ${ }^{2}$ Jérôme \\ Creuze ${ }^{3}$ Damien Alloyeau, ${ }^{1}$ Guillaume Wang, ${ }^{1}$ and Christian Ricolleau ${ }^{1}$ \\ ${ }^{1}$ Université Paris Diderot, Sorbonne Paris Cité, CNRS, \\ Laboratoire Matériaux et Phénomènes Quantiques, UMR 7162, 75013, Paris, France \\ ${ }^{2}$ Laboratoire d'Etude des Microstructures, ONERA-CNRS, BP 72, 92322 Châtillon Cedex, France \\ ${ }^{3}$ SP2M/ICMMO, Université Paris Sud-XI, UMR 8182, Bât. 410, F91405 Orsay Cedex, France
}

(Dated: December 8, 2017)

\begin{abstract}
We use in situ transmission electron microscopy to monitor in real-time the evaporation of gold, copper and bimetallic copper-gold nanoparticles at high temperature. Besides, we extend the Kelvin equation to two-component systems to predict the evaporation rates of spherical liquid mono- and bimetallic nanoparticles. By linking this macroscopic model to experimental TEM data, we determine the surface energies of pure gold, pure copper, $\mathrm{Cu}_{50} \mathrm{Au}_{50}$ and $\mathrm{Cu}_{25} \mathrm{Au}_{75}$ nanoparticles in the liquid state. Our model suggests that the surface energy varies linearly with the composition in liquid $\mathrm{Cu}-\mathrm{Au}$ nanoalloy, i.e. it follows a Vegard's rule-like dependence. To get atomic-scale insights into the thermodynamic properties of $\mathrm{Cu}-\mathrm{Au}$ alloys on the whole composition range, we perform Monte Carlo simulations employing $N$-body interatomic potentials. These simulations at microscopic level confirm the Vegard's rule-like behavior of the surface energy obtained from experiments combined with macroscopic modeling.
\end{abstract}

PACS numbers: 65.80.-g, 68.35.Md, 68.37.Lp

The surface energy of materials is a very important quantity for physics and chemistry since it drives a wide range of phenomena such as phase transition, crystal melting, evaporation and growth. For nanostructures, it is even more important as it controls not only their 3D morphology and their reactivity with respect to different environments, but it also plays an essential role in Ostwald ripening as well as in the growth rate of facets during nanoparticle (NP) coarsening. Thanks to the Wulff [1] and Wulff-Kaishew [2] relations giving the equilibrium shape of free and supported crystallites, respectively, surface energy ratios for low index facets of some metals were determined $[3,4]$. However, absolute surface energy determination is still rather difficult. For bulk and micron-size particles, several approaches were developed based on the temperature dependence of the 3D equilibrium shape, [5-8] on the detailed study of the $2 \mathrm{D}$ equilibrium morphology and its thermal fluctuations [9-14] or on a combination of the two previous approaches [15].

In the case of nanosized particles, Vermaak et al. showed that surface energy and the surface stress are equal for spherical NPs as long as their properties are similar to those of liquid droplets [16, 17]. This approximation is also valid if the strain rates can be considered as quasi-static at high temperatures. Several studies discussed the values of surface stress and surface energies and the origin of the discrepancies when they are different [18-22]. Another approach, based on the Kelvin equation [23] that relates equilibrium vapor pressure to surface curvature, was developed by Blackman et al. and Sambles et al. [24-26]. It derives a thermodynamic model of the evaporation of spherical liquid droplets and solid particles maintained at constant temperature. From the nonlinear relation between the time of complete evaporation and particle size, the mean surface energies of solid silver, [25], liquid lead [25] and liquid and solid gold [26] were successfully measured. Again, in NPs verifying Kelvin effect, a close approach that studies the linear relationship between the evaporation temperature and the inverse of the particle size was used to estimate the surface energies of silver [27] and $\mathrm{PbS}$ [28].

An alternative for determining surface energies of nanostructures is the direct computer simulation by using ab initio calculations [29-32] or semi-empirical potentials [33, 34]. In order to improve the accuracy of theoretical predictions, Medasani et al. have used both techniques to calculate the surface energy of pure NPs over a wide range of particle size [22]. However, the case of bimetallic NPs at given temperatures, which is much more complex, was not addressed.

In this letter, we extend Sambles approach [25] to determine experimentally the surface energies of monometallic copper and gold NPs as well as bimetallic $\mathrm{Cu}-\mathrm{Au}$ NPs by monitoring their evaporation at high temperatures by transmission electron microscopy (TEM) imaging. Adjustment of experimental TEM data by a macroscopic model based on an extended Kelvin equation suggests that the surface energy varies linearly with particle composition in liquid $\mathrm{Cu}-\mathrm{Au}$ nanoalloys. This Vegard's rule-like tendency is confirmed by simulations performed at atomic-scale on the whole composition range for $\mathrm{Cu}-\mathrm{Au}$ NPs in the liquid state.

TEM experiments were carried out using a JEOL JEM-ARM200F spherical aberration corrected electron 
microscope equipped with a cold field emission gun operated here at $80 \mathrm{kV}$ and an ultra-fast OneView camera (Gatan) for TEM imaging. In situ heating was performed using a slightly modified version of the Atmosphere TEM environmental gas cell from Protochips Inc. with heating capabilities up to $1000{ }^{\circ} \mathrm{C}$. Gold (Au), copper $(\mathrm{Cu})$ and bimetallic $\mathrm{Cu}_{50} \mathrm{Au}_{50}$ and $\mathrm{Cu}_{25} \mathrm{Au}_{75}$ nanoparticles were synthesized by alternated pulsed laser deposition (PLD) technique in a high vacuum chamber at $10^{-5} \mathrm{~Pa}$. Details on the experimental procedure for particle synthesis are published elsewhere [35]. Here, the nominal thickness of metal deposited for all three specimens was fixed at $2 \mathrm{~nm}$ and the temperature of the SiN substrate was held at $450{ }^{\circ} \mathrm{C}$ in order to obtain spherical shaped NPs with diameter below $10 \mathrm{~nm}$ at high temperatures. The composition of the bimetallic alloyed NPs were determined from lattice fringe spacing measurements in HRTEM images of single NPs at room temperature prior to their heating and evaporation at high temperatures. NP deposition was carried out directly on the 50-nm thick electron transparent silicon nitride membrane of the small heating E-chip that composes the gas cell. To enhance image contrast during TEM imaging of NPs at high temperatures, the SiN membrane of the larger E-chip of the gas cell was removed. The evaporation of copper, gold, $\mathrm{Cu}_{50} \mathrm{Au}_{50}$ and $\mathrm{Cu}_{25} \mathrm{Au}_{75}$ nanoalloys in vacuum were studied at $725^{\circ} \mathrm{C}$, $800{ }^{\circ} \mathrm{C}, 700{ }^{\circ} \mathrm{C}$ and $750{ }^{\circ} \mathrm{C}$ respectively. The three samples were heated up from room temperature to these temperatures at a rate of $1{ }^{\circ} \mathrm{C} / \mathrm{s}$. During the heat ramps and the evaporation process, the electron beam was turned off except for TEM imaging at regular intervals. In this way, we minimised unwanted electron beam effects like local increases in the temperature of the irradiated areas [36] and/or structural modifications of the NPs that could adversely accelerate particle evaporation. In general, NPs size and shape evolve during the annealing as a consequence of Ostwald ripening and/or coalescence. The crystalline state evolves as well with the temperature from solid to liquid to gas (melting / evaporation) or directly from solid to gas (sublimation). It is well known that the melting temperature of small NPs decreases as the particle size decreases [37, 38]. Here, we focus on the evaporation kinetics of NPs with radius $\phi<3 \mathrm{~nm}$ which are all in the liquid state at high temperature $\left(>700{ }^{\circ} \mathrm{C}\right)$ as shown by their uniform image contrast and the absence of lattice fringes in their TEM images (see discussion in Sec. I of the Supplemental Material [39]). We followed the variation of the NPs size as a function of time at a given temperature. For the three systems, there is a clear decrease of the particle radius with time as predicted by the Kelvin equation. [25]. When the NPs are imaged under continuous illumination, TEM observations show substantial particle sintering via dynamic diffusion and Ostwald ripening (see Fig. 2s of the Supplemental Material [39]). These transformations are characteristic of electron beam-induced effects as they are observed even in the absence of any heating. Under intermittent electron illumination, particle mobility on the heated SiN support is suppressed which is a strong indication that the effect of the electron beam is largely minimized and that the evaporation of NPs observed experimentally is activated primarily by the thermal heating of the SiN support.

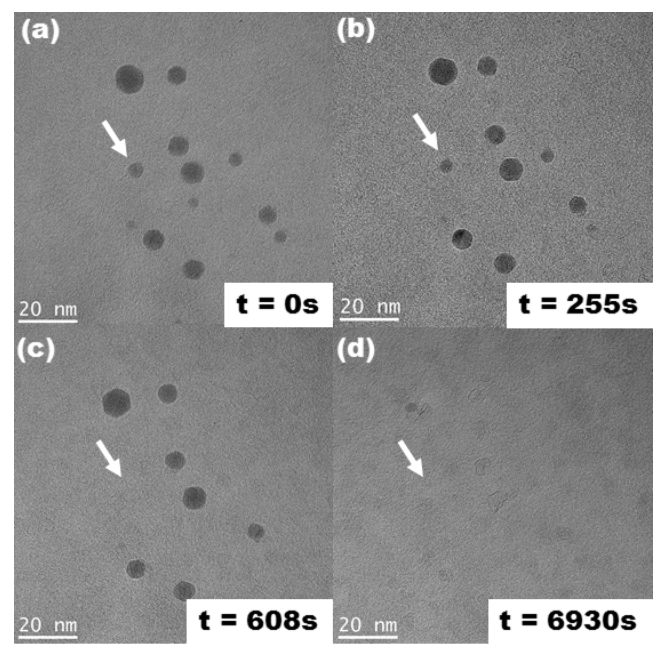

(e)

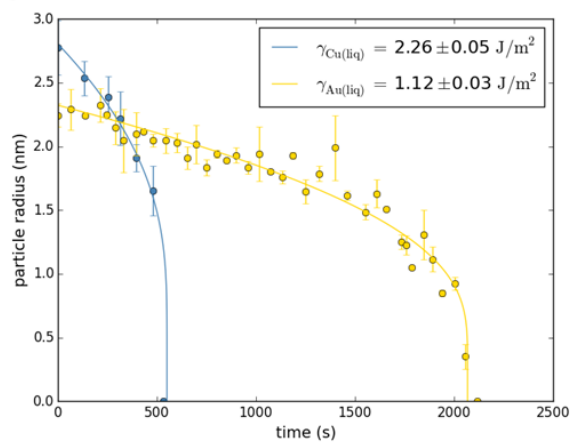

FIG. 1. (a)-(d) In situ TEM monitoring of the evaporation of a set of $\mathrm{Cu}$ NPs at $725{ }^{\circ} \mathrm{C}$ (e) Evaporation curves of a $\mathrm{Au} \mathrm{NP}$ at $800{ }^{\circ} \mathrm{C}$ and a $\mathrm{Cu} \mathrm{NP}$ (indicated by a white arrow in (a)-(d)) at $725{ }^{\circ} \mathrm{C}$. Circles correspond to the experimental data adjusted by the evaporation of single-component system obtained from Kelvin equation (full line). Values of the surface energy extracted from the fitting of experimental data are given in insert.

Figures 1(a)-(d) compare the TEM images of a set of $\mathrm{Cu}$ NPs at $725{ }^{\circ} \mathrm{C}$ at different times over a $115.5 \mathrm{~min}-$ utes period. These TEM images show that the NPs are completely evaporated over this period. The full followup of particle evaporation is shown in Fig. 3S of the Supplemental Material [39]. The evaporation curves of a pure $\mathrm{Au} \mathrm{NP}(\bullet)$ and a pure $\mathrm{Cu} \mathrm{NP}(\bullet)$ is presented in Fig. 1(e). The uncertainties in the determination of particle radius were estimated by measuring the latter along two perpendicular directions in the TEM images. The evaporation time $t$ of a monometallic liquid NP of radius 
$r$ at a temperature $T$ is given by (see Sec. IV and V of the Supplemental Material [39]):

$$
t(r)=\frac{B}{A}\left[E_{0}\left(\frac{B}{r}\right)-E_{1}\left(\frac{B}{r}\right)\right]
$$

with:

$$
A=\frac{P_{\infty}}{\rho_{l}}\left(\frac{M_{l}}{2 \pi R T}\right)^{\frac{1}{2}} ; B=\frac{2 \gamma M_{l}}{R T \rho_{l}}
$$

and

$$
E_{0}(x)=\frac{\exp (-x)}{x} ; E_{1(x)}=\int_{x}^{\infty} \frac{\exp (-y)}{y} d y
$$

where $\gamma$ is the surface energy of the metal, $M_{l}$ the molar mass of the liquid phase, $\rho_{l}$ its density, $P_{\infty}$ the equilibrium vapor pressure over a flat surface of the liquid and $R$ the ideal gas constant. By fitting the experimental data in Fig. 1(c) to the Kelvin model according to Eq. 1, we extract the surface energy for liquid gold and copper NPs. We obtain a mean surface energy of $0.93 \pm 0.23$ $\mathrm{J} . \mathrm{m}^{-2}$ and $2.03 \pm 0.16 \mathrm{~J} . \mathrm{m}^{-2}$. These surface energies are averaged over four $\mathrm{Au}$ and three $\mathrm{Cu}$ NPs respectively.

To describe the evaporation of bimetallic $\mathrm{Cu}-\mathrm{Au} \mathrm{NPs}$, we extend Sambles et al. [25] approach to spherical liquid bimetallic $A-B$ particle under the following hypotheses. We assume that the composition of the liquid phase does not vary during the evaporation process. As a result, the compositions of liquid and vapor phases are always similar and equal to the initial composition of the liquid NP. Moreover, the bimetallic metal vapor in equilibrium with the liquid NP is considered as a mixture of ideal gases $A$ and $B$. Finally, we consider that the bimetallic liquid phase behaves like an ideal liquid mixture with the partial pressure of each component following Raoult's law. As we will see later, the hypothese of an ideal liquid mixture is validated by atomic scale modeling. Under these conditions, the Kelvin equation of a bimetallic $A-B$ alloy is given by (see Sec. V of the Supplemental Material [39]):

$$
\frac{P_{r}}{P_{\infty}}=\exp \left(\frac{2 \gamma\left(\alpha M_{A}+M_{B}\right)}{(\alpha+1) R T \rho_{A, B} r}\right)
$$

where $P_{\infty}$ is the total vapor pressure of the twocomponent gas system in equilibrium with a semi-infinite bimetallic $A-B$ planar surface and $P_{r}$, that of the gas in equilibrium with the curved surface of a spherical bimetallic liquid droplet of radius $r$ at temperature $T$. $\alpha$ $=n_{A} / n_{B}$ is the molar ratio, $M_{A}$ and $M_{B}$ are the molecular weights of the two metals and $\rho_{A, B}$ is the liquid density of the mixed compound. From the extended Kelvin equation, the evaporation time $t$ of a bimetallic alloyed $\mathrm{NP}$ with radius $r$ is also defined through Eq.(1) but with (see Sec. V of the Supplemental Material [39]):

$$
\begin{aligned}
A & =\frac{P_{\infty}}{(1+\alpha)}\left[\frac{\alpha}{\rho_{A}}\left(\frac{M_{A}}{2 \pi R T}\right)^{\frac{1}{2}}+\frac{1}{\rho_{B}}\left(\frac{M_{b}}{2 \pi R T}\right)^{\frac{1}{2}}\right] \\
B & =\frac{2 \gamma\left(\alpha M_{A}+M_{B}\right)}{R T(1+\alpha) \rho_{A, B}}
\end{aligned}
$$

Figure 2 shows the evaporation curves at $700{ }^{\circ} \mathrm{C}$ of five $\mathrm{Cu}_{50} \mathrm{Au}_{50}$ NPs with initial radii less than $3 \mathrm{~nm}$. Adjustment of experimental evaporation curves of seven bimetallic NPs by the model based on the extended Kelvin equation gives a mean surface energy for liquid $\mathrm{Cu}_{50} \mathrm{Au}_{50}$ nanoalloy equal to $1.530 \pm 0.076 \mathrm{~J}^{\mathrm{m}}{ }^{-2}$. This value is nearly the average between the experimentallydetermined surface energies of liquid copper and liquid gold. For $\mathrm{Cu}_{25} \mathrm{Au}_{75} \mathrm{NPs}$, a mean surface energy of 1.230 $\pm 0.047 \mathrm{~J} / \mathrm{m}^{2}$ is obtained by analyzing six NPs with radii below $3 \mathrm{~nm}$ (Sec. VI of the Supplemental Material [39] for the corresponding evaporation curves). Thus, determination of the absolute values of the surface energy of the monometallic and bimetallic NPs suggests that the surface energy for $\mathrm{Cu}-\mathrm{Au}$ nanoalloy follows a Vegard's rule-like behavior. The exact shape of the NPs are not known as TEM images only give their two-dimensional projection perpendicular to the substrate. However, the NPs are most probably greater than a hemisphere such that the experimentally-measured radii projected in the plane of the substrate are equal to their radius of curvature which appears in the Kelvin equation. Indeed, $\mathrm{Au}$, $\mathrm{Cu}$ and $\mathrm{Cu}-\mathrm{Au}$ NPs are loosely bound to the SiN support due to the hydrophobic nature of the latter. This hydrophobicity increases when the SiN film upon heating as residual moisture are taken off. As metal adhesion is reduced, the contact angles of $\mathrm{Au}, \mathrm{Cu}$ and $\mathrm{Cu}-\mathrm{Au} \mathrm{NPs}$ are expected to be close to $180^{\circ}$ above $700{ }^{\circ} \mathrm{C}$. On $\mathrm{Si}_{3} \mathrm{~N}_{4}$ film, $\mathrm{Cu}$ shows low wettability with a contact angle of about $130^{\circ}$ above $1000{ }^{\circ} \mathrm{C}$ [54]. When the truncation is greater or equal to a hemisphere as in our experiments, the average change in the rate of evaporation is of the order of $6 \%$ with are within the uncertainties of the surface energies determined by our model.

To get insight at atomic-scale and investigate the thermodynamic properties of $\mathrm{Cu}-\mathrm{Au}$ alloys, we perform Monte Carlo (MC) simulations in the canonical ensemble (with fixed concentrations). This approach, unlike others found in the literature [55-57], enables thermodynamic study over a broad-spectrum of temperatures $(0<T<2000 \mathrm{~K})$ on the whole composition range at the atomic scale. In this context, we develop a specific $\mathrm{N}$-body interatomic potential derived from the second moment approximation of the tight-binding scheme(TBSMA) $[42,43,58]$ perfectly adapted to deal with large systems ( 1000-10000 atoms) similar to those observed experimentally. The accuracy of TB-SMA models depends critically on the validity of the database to which the parameters of the potentials are fitted. Here, we sum- 


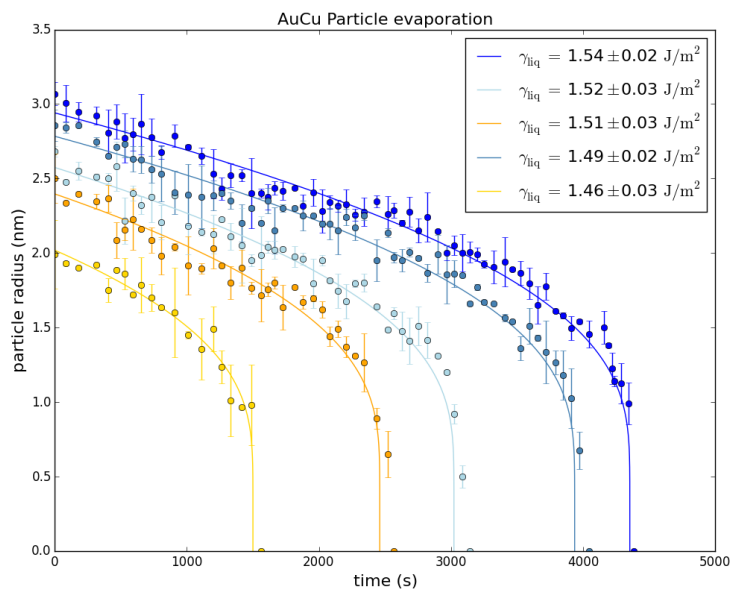

FIG. 2. Evaporation curves of liquid $\mathrm{Cu}_{0.5} \mathrm{Au}_{0.5} \mathrm{NPs}$ at 700 ${ }^{\circ} \mathrm{C}$. Experimental data (circles) are modeled by the extended Kelvin equation for two-component system (full line).

marize only the crucial points of the TB-SMA potential and refer the reader to Sec. VII of the Supplemental Material [39] for more details. For pure elements, they are obtained by fitting on several bulk physical quantities (lattice parameters, cohesive energies and elastic constants). Note that fitting the TB-SMA parameters to experimental cohesive energies leads to an underestimation $(\sim$ a factor 2$)$ of the surface energies since it is well known that such potentials are not always adapted to describe physical properties from bulk to surface [59]. Nevertheless, these deviations do not prevent us to describe qualitatively structural properties of $\mathrm{Cu}-\mathrm{Au}$ nanoalloys as we will see in the following. Improving the accuracy usually implies increasing the number of parameters, which can blur the physical transparency of the model [60-62]. Besides, we can notice that the Au surface energy calculated is lower than the one of $\mathrm{Cu}$, in agreement with DFT calculations, which is in favor of the Au surface segregation, as already discussed in [55]. For the $\mathrm{Cu}-$ $\mathrm{Au}$ system, the potential has been fitted both to DFT calculations [63] and to the experimental bulk phase diagram [47], in particular to experimental order/disorder transition temperatures. This TB-SMA potential is then implemented in a MC code to relax the structures at finite temperatures [49] (see Sec. VIII of the Supplemental Material [39]).

As a first step, we focus on liquid NPs to investigate their structural properties (physical and chemical states) by using canonical MC simulations at different temperatures. A typical example is presented in Fig. 3 where the starting configuration is a cuboctahedron containing 2507 atoms and has size of $4 \mathrm{~nm}$ comparable to those studied experimentally. Interestingly, a visual inspection shows no segregation effect in the liquid state. This is confirmed in a more quantitative way with the analysis
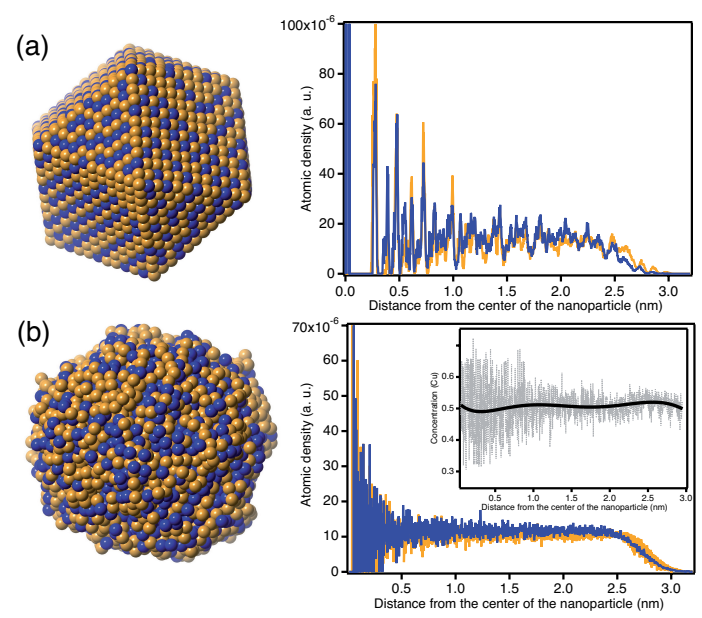

FIG. 3. Properties of $\mathrm{Cu}_{50} \mathrm{Au}_{50}$ NPs calculated for MC runs at (a) $300 \mathrm{~K}$ and (b) $1500 \mathrm{~K}$. (left) Equilibrium configurations (right) Density and composition profiles along the radius of the NP (orange: $\mathrm{Au}$, blue: $\mathrm{Cu}$ ).

of the density profiles along the radius of the NPs depicted in Fig. 3 and whatever the concentration and the size of the NPs (see Sec. VIII in the Supplemental Material [39]). The calculation of the surface energy with the composition is therefore relevant since a disordered alloy is formed at the surface of the NP. Actually, the same phenomena can be observed for a slab (see Figure 7S(a) in Supplemental Material [39]). As a result, a slab will be considered in the following since the definition of $\gamma$ for alloys is straightforward in this case. Indeed, surfaces can be created by dividing an infinite crystal into two parts. The energy needed to cut the bonds and bring the two resulting parts to infinity determines the surface energy :

$$
\gamma=\frac{E_{\text {slab }}-E_{\text {bulk }}}{2 S},
$$

where $S$ is the surface area. $E_{\text {slab }}$ and $E_{b u l k}$ represent the total energies of the slab and the bulk containing both $N$ atoms at different concentrations, respectively. Here, we use Monte Carlo simulations to calculate these quantities at temperatures where the system is liquid. In the present calculations, the surface energy converges properly for large enough slabs. More precisely, we consider a slab size equal to $36.14 \times 36.51 \times 36.90 \AA^{3}$ containing 2520 atoms. As shown in Fig. 4 where two temperatures are considered, surface energies vary linearly with the composition of the alloy following the theoretical Vegard's rule-like behavior. Unexpectedly, this is not the case for $E_{\text {slab }}$ and $E_{\text {bulk }}$ (see Figure 7S(b) in Suppplementary Material [39]). Moreover, by taking into account the factor 2 discussed previously, experimental and theoretical data are in very good agreement. Therefore, it can be concluded that our model based on the Kelvin equation is robust to determine the surface energy of an alloy 
for all concentrations and not only for the equiatomic composition.

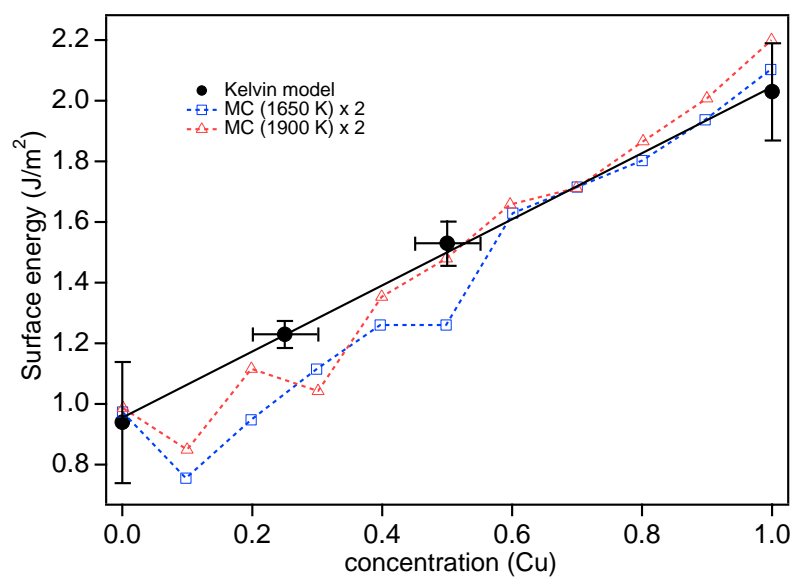

FIG. 4. Variation of the surface energy of $\mathrm{Au}_{1-x} \mathrm{Cu}_{x}$ systems in the liquid state as a function of the $\mathrm{Cu}$ composition. The full black line is a linear fit of experimental data.

In summary, by combining in situ TEM observations of the thermally-activated evaporation of $\mathrm{Au}, \mathrm{Cu}$ and $\mathrm{Cu}_{50} \mathrm{Au}_{50}$ NPs and an extended Kelvin equation for twocomponent systems, we have determined the surface energy of these metals in the liquid phase. Our results show that the surface energy of liquid bimetallic $\mathrm{Cu}-\mathrm{Au}$ nanoalloys follows a Vegard's rule-like dependence. This dependence was confirmed by Monte Carlo simulations using an optimized tight binding TB-SMA potential that described very well the thermodynamic properties of the $\mathrm{Cu}-\mathrm{Au}$ nanoalloy. This work constitutes a major step in the determination of surface energy of alloyed nanoparticles experimentally which is still a challenge.

The authors acknowledge the Région Ile-de-France for convention SESAME E1845 for the support of the JEOL JEM-ARM200F installed at Paris Diderot University. J.C and H.A. thank F. Ducastelle and B. Legrand for fruitful discussions.

[1] G. Wulff, Z. Kristallogr. 34, 449 (1901).

[2] R. Kaishew, Comm. Bulg. Acad. Sci. 1, 100 (1950).

[3] J. C. Heyraud and J. J. Métois, Acta Metallurgica 28, 1789 (1980).

[4] J. C. Heyraud and J. J. Métois, Surf. Sci. 128, 334 (1983).

[5] H. P. Bonzel and A. Emundts, Phys. Rev. Lett. 84, 5804 (2000).

[6] H. Bonzel, Prog. Surf. Sci. 67, 45 (2001).

[7] H. Bonzel, Interf. Sci. 9, 21 (2001).

[8] H. Bonzel, Phys. Rep. 385, 1 (2003).

[9] N. C. Bartelt, R. M. Tromp, and E. D. Williams, Phys. Rev. Lett. 73, 1656 (1994).
[10] D. C. Schlößer, L. K. Verheij, G. Rosenfeld, and G. Comsa, Phys. Rev. Lett. 82, 3843 (1999).

[11] G. Schulze Icking-Konert, M. Giesen, and H. Ibach, Phys. Rev. Lett. 83, 3880 (1999).

[12] M. Giesen, C. Steimer, and H. Ibach, Surf. Sci. 471, 80 (2001).

[13] S. Kodambaka, V. Petrova, S. V. Khare, D. D. Johnson, I. Petrov, and J. E. Greene, Phys. Rev. Lett. 88, 146101 (2002).

[14] S. Kodambaka, S. V. Khare, V. Petrova, D. D. Johnson, I. Petrov, and J. E. Greene, Phys. Rev. B 67, 035409 (2003).

[15] J. J. Métois and P. Müller, Surf. Sci. 548, 13 (2004).

[16] J. S. Vermaak, C. W. Mays, and D. Kuhlmann-Wilsdorf, Surf. Sci. 12, 128 (1968).

[17] C. W. Mays, J. S. Vermaak, and D. Kuhlmann-Wilsdorf, Surf. Sci. 12, 133 (1968).

[18] H. J. Wasserman and J. S. Vermaak, Surf. Sci. 22, 164 (1970)

[19] P. A. Montano, W. Schulze, B. Tesche, G. K. Shenoy, and T. I. Morrison, Phys. Rev. B 30, 672 (1984).

[20] C. Solliard and M. Flueli, Surf. Sci. 156, 487 (1985).

[21] H. Hofmeister, S. Thiel, M. Dubiel, and E. Schurig, Appl. Phys. Lett. 70, 1694 (1997).

[22] B. Medasani, Y. H. Park, and I. Vasiliev, Phys. Rev. B 75, 235436 (2007).

[23] L. M. Skinner and J. R. Sambles, Aerosol Science 3, 199 (1972).

[24] M. Blackman, N. Lisgarten, and L. Skinner, Nature 217, 1245 (1968).

[25] J. R. Sambles, L. M. Skinner, and N. D. Lisgarten, Proc. Roy. Soc. Lond. A 318, 507 (1970).

[26] J. Sambles, Proc. Roy. Soc. Lond. A 324, 339 (1971).

[27] K. K. Nanda, A. Maisels, F. E. Kruis, H. Fissan, and S. Stappert, Phys. Rev. Lett. 91, 106102 (2003).

[28] K. K. Nanda, F. E. Kruis, and H. Fissan, Phys. Rev. Lett. 89, 256103 (2002).

[29] M. Methfessel, D. Hennig, and M. Scheffler, Phys. Rev. B 46, 4816 (1992).

[30] H. L. Skriver and N. M. Rosengaard, Phys. Rev. B 46, 7157 (1992).

[31] J. Kollár, L. Vitos, and H. L. Skriver, Phys. Rev. B 49, 11288 (1994).

[32] L. Vitos, A. V. Ruban, H. L. Skriver, and J. Kollár, Surf. Sci. 411, 186 (1998).

[33] M. S. Daw and M. I. Baskes, Phys. Rev. B 29, 6443 (1984).

[34] A. M. Rodríguez, G. Bozzolo, and J. Ferrante, Surf. Sci. 289, 100 (1993).

[35] H. Prunier, J. Nelayah, C. Ricolleau, G. Wang, S. Nowak, A.-F. Lamic-Humblot, and D. Alloyeau, Phys. Chem. Chem. Phys. 17, 28339 (2015).

[36] M. A. Asoro, D. Kovar, and P. Ferreira, ACS Nano 7, 7844 (2013).

[37] P. Buffat and J.-P. Borel, Phys. Rev. A 13, 2287 (1976).

[38] T. Castro, R. Reifenberger, E. Choi, and R. P. Andres, Phys. Rev. B 42, 8548 (1990).

[39] See Supplemental Material at [URL] for additional TEM data, the generalization of Kelvin equation to a twocomponent sytem and derivation of the evaporation kinetic for such system, details on the second moment approximation interatomic potential and Monte Carlo simulations which includes Refs [25, 40-53].

[40] P. G. de Gennes, F. Brochard-Wyart, and D. Quéré, 
Capillarity and Wetting Phenomena (Springer, 2004) pp. 7-8.

[41] G. kaptay, G. Csicsovszki, and M. S. Yaghmaee, Materials Science Forum 414-415, 235 (2003).

[42] V. Rosato, M. Guillopé, and B. Legrand, Philosophical Magazine A 59, 321 (1989).

[43] J. Creuze, I. Braems, F. Berthier, C. Mottet, G. Tréglia, and B. Legrand, Phys. Rev. B 78, 075413 (2008).

[44] C. Kittel, Introduction to Solid State Physics, 7th ed. (Wiley, New York, 1995).

[45] G. Simmons and H. Wang, Single crystal elastic constants and calculated agregates properties (MIT, Cambridge, 1971).

[46] A. R. Miedema and A. K. Niessen, Calphad 7 (1983).

[47] H. Okamoto, D. J. Chakrabarti, D. E. Laughlin, and T. B. Massalski, Bull. of Alloy Phase Diagrams 8, 454 (1987).

[48] R. Hultgren, P. D. Desai, D. T. Hawkins, M. Gleiser, and K. K. Kelly, Selected Values of the Thermodynamic Properties of Binary Alloys (Wiley, New York, 1973), 1973).

[49] D. Frenkel and B. Smith, Understanding Molecular Simulation (Academic Press: London, 2002).

[50] Y. Yang, M. Asta, and B. B. Laird, Phys. Rev. Lett. 110, 096102 (2013).
[51] A. Statt, P. Virnau, and K. Binder, Phys. Rev. Lett. 114, 026101 (2015).

[52] F. Ducastelle, "Nanoalloys: Synthesis, structure and properties," (Springer-Verlag, London, 2012) Chap. Chemical Order and Disorder in Alloys, pp. 197-225.

[53] F. Calvo, Phys. Chem. Chem. Phys. 17, 27922 (2015).

[54] M. K. M. Naka and I. Okamoto, Journal of Material Science Letters 6, 965 (1987).

[55] G. Guisbiers, S. Mejia-Rosales, S. Khanal, F. RuizZepeda, R. L. Whetten, and M. José-Yacaman, Nano Lett. 14, 6718 (2014).

[56] F. M. Takrori and A. Ayyad, Appl. Surf. Sci. 401, 65 (2017).

[57] V. Ozoliņš, C. Wolverton, and A. Zunger, Phys. Rev. B 57, 6427 (1998).

[58] F. Ducastelle, J. Phys. (Paris) 31, 1055 (1970).

[59] M. S. S. Brooks and B. Johansson, J. Phys. F: Met. Phys. 13, L197 (1983).

[60] J. Guevara, A. M. Llois, and M. Weissmann, Phys. Rev. B 52, 11509 (1995).

[61] C. Barreteau, F. Raouafi, M. C. Desjonquères, and D. Spanjaard, Surf. Sci. 519, 15 (2002).

[62] C. Goyhenex, Surf. Sci. 606, 325 (2012).

[63] Y. Zhang, G. Kresse, and C. Wolverton, Phys. Rev. Lett. 112, 075502 (2014). 